\title{
Advances on Smart Object Management
}

\author{
Kostas Pentikousis • Ramón Agüero • \\ Andreas Timm-Giel $\cdot$ Susana Sargento
}

Published online: 9 February 2014

(C) Springer Science+Business Media New York 2014

\section{Special issue introduction}

The first part of this issue features four papers that discuss advanced management techniques for Smart Objects. The socalled Internet of Things (IoT) is one of the cornerstones of the Future Internet. One illustrative example of the relevance of IoT in future network development is its growing adoption within the smart city paradigm, as a means to provide enhanced citizen services. In this sense, basic IoT technology is no longer at the purely academic research level, but is starting to be integrated to the the fabric of our daily activities.

One of the elements that are required to support the successful deployment of this type of architectures is having the appropriate management mechanisms in place. The call for papers for this special issue was a result of a dedicated workshop on the management of smart objects. The workshop was collocated with the 4th International Conference on Mobile Networks and Management (MONAMI 2012), which was organized in close collaboration with the Technical University of Hamburg in September 2012 (see www.mon-ami.org/2012). The four papers which were accepted for publication in this special

\author{
K. Pentikousis $(\bowtie)$ \\ European Center for Information and Communication Technologies \\ (EICT), EUREF Campus Haus 13, Torgauer Straße 12-15, \\ 10829 Berlin, Germany \\ e-mail: k.pentikousis@eict.de \\ R. Agüero \\ University of Cantabria, Santander, Spain \\ e-mail: ramon@tlmat.unican.es \\ A. Timm-Giel \\ Hamburg University of Technology, Hamburg, Germany \\ e-mail: timm-giel@tuhh.de \\ S. Sargento \\ University of Aveiro, Aveiro, Portugal \\ e-mail: susana@ua.pt
}

issue deal with management architecture alternatives, service development frameworks, security challenges, and the role that contextual information has in the Internet of Things. All in all, they provide a comprehensive outlook on some of the problems that need to be addressed for this type of deployments. It is worth highlighting that three of the works do really exemplify the need of real deployments and employ implementation over existing technologies to assess the feasibility of their proposed architectures, frameworks and techniques.

In the first paper, JaeSeung Song et al. review some of the architectural choices for M2M networks. They start from the challenges that need to be addressed and discuss how the various standardization bodies (for instance, ETSI and 3GPP) are tackling them. They then present their approach for some of the technical functionalities required to control and manage M2M networks. Finally the authors describe a realization of a subset of the aforementioned techniques over a real testbed, using the service model proposed by the SENSEI European project. The authors use three performance indicators to assess the goodness of the techniques, namely the stability, the scalability and the robustness. The results, that are as well included within the CAMPUS 21 project, show that the proposed scheme can run on relatively low-memory devices, making it very attractive for realworld IoT deployments.

One key success factor for IoT is the possibility to enable fast service creation, which is open to the general public, so that a user does not need to be an expert to be able to create his/her own service. In order to address these challenges, Sylvain Cherrier et al. propose, in the second paper of this special issue, a framework to deploy services to be used over IoT based on the composition of behaviours. They go beyond their previous proposal, D-Lite, which was based on Finite State Transducers, and propose a simpler way of modeling the interactions between IoT components. The $\mathrm{BeC} 3$ architecture also allows the exploitation of available modules and 
components. The paper presents an implementation that assesses the possibilities that are brought forward by their proposal.

If there is one particular characteristic of IoT that sets it apart is the requirement for delivering a massive amount of contextual information. At the time of this writing, the Big Data paradigm is taking hold of the strategies for future development at all global industrial players, and it is not unrealistic to assume that it will become commonplace in the short-term. One of the potential benefits that might be provided by IoT massive deployments is precisely the amount of information that they might be able to provide. Clearly there must be some tradeoff between the amount of data to be acquired/processed/delivered and the cost to do that. The third paper proposes a four-layer scheme to alleviate this problem and to allow applications that are executed over the IoT substrate to benefit from the potential information at hand without a strong impact over the operational lifetime of devices, or the required communication overhead. Stefan Forsström and Theo Kanter use a proof-of-concept prototype to assess the feasibility of their proposal, which is based on limiting the information exchange considering its relevance.

Finally, an aspect that must be carefully addressed in order to promote real deployment of IoT is security. Sometimes security is left as a feature that is taken for granted, and it does not really receive enough attention early on in the system design process. In the fourth paper of this special issue, Swaminathan Sankararaman et al. propose a method to systematically place jammers within a particular network deployment. This would allow to have security in place, without the burden to add complex and expensive ciphering schemes. The paper provides a thorough mathematical formulation of the problem while the proposed solution is assessed through simulation.

\section{Guest editor biographies}

Kostas Pentikousis is the Head of IT Infrastructure at EICT $\mathrm{GmbH}$, a public-private partnership which acts as a trusted third party and an international platform for interdisciplinary collaborative research. Prior to joining EICT, he was a senior research engineer at Huawei Technologies in Berlin, Germany and a standards delegate to IETF. From 2005 to 2009 he was a senior research scientist with VTT Technical Research Centre of Finland. He earned his Bachelor's degree in informatics (1996) from Aristotle University of Thessaloniki, Greece, and his Master's (2000) and doctoral degrees (2004) in computer science from the State University of New York at Stony Brook. Dr. Pentikousis conducts research in Internet protocols and network architecture,with contributions ranging from system design and implementation to performance evaluation and standardization.
Ramón Agüero received a degree in Telecommunications Engineering from the University of Cantabria in 2001 and the $\mathrm{PhD}$ in 2008. He is currently an Associate Professor at the Communications Engineering Department at that university. He has participated in several collaborative research projects and his research focuses on future network architectures, especially regarding the (wireless) access part of the network. $\mathrm{He}$ is also interested on multi-hop (mesh) networks and device-to-device communications. He has published more than 100 technical papers in such areas and he is a regular TPC member and reviewer on various related conferences and journals.

Andreas Timm-Giel (www.tuhh.de/comnets) is full professor and head of the Institute of Communication Networks (ComNets) at Hamburg University of Technology (TUHH). Furthermore, he is coordinating TUHH's research center on Mobile Sensor and Data Networks (SOMSED) and is deputy head of the School of Electrical Engineering, Computer Science and Mathematics. From 2002 till 2009 he was with the Communication Networks group of Bremen University as senior researcher and lecturer. He was leading several industrial, national and EC funded research projects and from 2006 he was additionally directing the interdisciplinary activity "Adaptive Communications" of TZI (Center of Computing and Communication Technologies). Before, he was with MediaMobil GmbH and its Joint Venture M2SAT Ltd. for three years, acting as Technical Project Leader and Manager Network Operations. He received his PhD (Dr.-Ing) and Master Degree (Dipl.-Ing) from Bremen University in 1999 and 1994 respectively. Here he lead a group on Mobile and Satellite Communications and was involved in several EU funded projects for more than 5 years. His research interests are mobile and wireless communications, sensor networks and the Future Internet. Prof. Timm-Giel is author or coauthor more than 100 peer-reviewed publications in journals and on international conferences. He is frequent reviewer and TPC member for international conferences and journals and is Member of IEEE and VDE/ITG. He is speaker of the ITG group 5.2.1 "System Architectures and Traffic Engineering" and member of the editorial board of the Elsevier's International Journal of Electronics and Communications.

Susana Sargento (http://www.av.it.pt/ssargento) received her Ph.D. in 2003 in Electrical Engineering. She joined the Department of Computer Science of the University of Porto in September 2002, and is in the Universidade de Aveiro and the Instituto de Telecomunicações since February 2004, where she is leading the Network Architectures and Protocols (NAP) group (http://nap.av.it.pt). She is also a Guest Faculty of the Department of Electrical and Computer Engineering from Carnegie Mellon University, USA, since August 2008, where she performed Faculty Exchange in 2010/2011. She has been involved in several national and European projects, taking leaderships of several activities in the projects, such 
as the QoS and ad-hoc networks integration activity in the FP6 IST-Daidalos Project. She has been recently involved in several FP7 projects (4WARD, Euro-NF, C-Cast, WIP, Daidalos, C-Mobile), national projects, and Carnegie Mellon Portugal research projects (DRIVE-IN with the Carnegie Melon University). She has been TPC-Chair and organizing several conferences, such as MONAMI'11, NGI'09, IEEE ISCC'07, NTMS'12, IEEE FEDNET (with IEEE NOMS'12), IEEE
IoT-SoS in IEEE WoWMoM 2013 and ISCC 2014. She has also been a reviewer of numerous international conferences and journals, such as IEEE Wireless Communications, IEEE Networks, IEEE Communications. Her main research interests are in the areas of Next Generation and Future Networks, more specifically QoS, mobility, self- and cognitive networks. She regularly acts as an Expert for European Research Programmes. 